\title{
The acute effect of the antioxidant drug "u-74389g" on platelet distribution width during hypoxia reoxygenation injury in rats
}

\begin{abstract}
Background: Platelet activation causes morphologic changes of platelets, including both the spherical shape and pseudopodia formation increasing platelet distribution width (PDW) ascribed to platelet anisocytosis. Furthermore, coagulation is activated in vaso-occlusive situations, increasing PDW. Early diagnosis of progressive activation of coagulation or any factor declining PDW levels, can prevent these thromboembolic diseases successfully. The probable beneficial effect of the molecule "U-74389" was studied hematologically using blood mean platelet distribution width PDW) levels.

Methods: 40 rats of mean weight $231.875 \mathrm{~g}$ were used in the study. PDW levels were measured at $60 \mathrm{~min}$ of reoxygenation (groups $\mathrm{A}$ and $\mathrm{C}$ ) and at $120 \mathrm{~min}$ of reoxygenation (groups B and D) with administration of the drug U-74389G in groups C and D.

Results: U-74389G administration kept significantly increased the PDW levels by $1.79 \% \pm 0.76 \%$ ( $\mathrm{p}=0.0314)$. Reoxygenation time non significantly declined the PDW levels by $0.745 \% \pm 0.81 \%(\mathrm{p}=0.3280)$. However, $\mathrm{U}-74389 \mathrm{G}$ administration and reoxygenation time together kept significantly increased the PDW levels by $0.96 \% \pm 0.46 \%(\mathrm{p}=0.0396)$.

Conclusion: U-74389G administration whether it interacted or not with reoxygenation time borderline declines from significant to non significant the PDW levels, within short-time study period of 2 hours. This finding has huge clinical interest in coagulopathy prevention or reversion.
\end{abstract}

Keywords: Hypoxia, U-74389G, Platelet distribution width, Reoxygenation
Volume 3 Issue 6 - 2015

\author{
Constantinos Tsompos,' C Panoulis, ${ }^{2} \mathrm{~K}$ \\ Toutouzas, ${ }^{3}$ G Zografos, ${ }^{4}$ A Papalois ${ }^{5}$ \\ 'Department of Obstetrics \& gynecology, Mesologi County \\ Hospital, Greece \\ ${ }^{2}$ Department of Obstetrics \& gynecology, Aretaieion Hospital, \\ Athens University, Greece \\ ${ }^{3}$ Department of Surgery, Ippokrateion General Hospital, Athens \\ University, Greece \\ ${ }^{4}$ Department of surgery, Ippokrateion General Hospital,Athens \\ University, Greece \\ ${ }^{5}$ Director Biologist, Exprerimental Research Center ELPEN \\ Pharmaceuticals, S.A. Inc., Co., Greece
}

\begin{abstract}
Correspondence: Tsompos Constantinos, Department of Obstetrics \& Gynecology, Mesologi County Hospital, Nafpaktou street, Mesologi 32, Etoloakarnania, Greece,T,Tel 0030263 I 360237, 00306946674264, Fax 3.02107E+I I, Email constantinostsompos@yahoo.com
\end{abstract}

Received: November 02, 2015 | Published: December 23, 2015

\section{Introduction}

Tissue hypoxia- reoxygenation (HR) are seriously implicate the adjacent organs and systems impairment. The use of U-74389G in HR has been a challenge for many years. However, although the progress was significant, several practical questions have not clarified.

They include:

1. the potency size of $\mathrm{U}-74389 \mathrm{G}$

2. the administration timing

3. the optimal dosage of U-74389G

The promising prevention of $\mathrm{U}-74389 \mathrm{G}$ in tissue homeostasis has been tested in several HR studies. The chemical type of U-74389G is 21-(4-(2,6-di-1-pyrrolidinyl-4-pyrimidinyl)-1-piperazinylpregna$1,4,9(11)$-triene-3,20-dione maleate salt. It is about an antioxidant agent which prevents the peroxidation of both arachidonic acid and iron-dependent lipids. ${ }^{1}$ It neutralizes the HR oxidative injury of many animal organs. This agent has been proved to prevent permeability alterations in brain endovascular cells monolayers. ${ }^{2}$ A more generalized numeric evaluation of the U-74389G efficacy was tried to be outcome by a meta-analysis of 13 published seric variables, for the same experimental endpoints (Table 1) ${ }^{3-5}$ ). This study is accordant with a recent trend of testing many other similar antioxidant molecules. ${ }^{6}$

Thromboembolic diseases are among the major cause of mortality in developed countries. Early diagnosis of progressive activation of coagulation can help manage these diseases successfully. Platelet activation causes morphologic changes of platelets, including both the spherical shape and pseudopodia formation. Platelets with increased number and size of pseudopodia differ in size, possibly affecting PDW. Mean values of PDW were significantly higher in patients with asserted platelet activation. Platelets change in shape during activation in order to obtain a larger surface. Their shape changes from discoid to spherical. Pseudopodia are formed as well. Therefore activated platelets seem larger. PDW was higher in subgroups of certain platelet activation. The increase of PDW was ascribed to platelet anisocytosis, which results from pseudopodia formation. However, coagulation is activated in vaso-occlusive situations, possibly causing this increase of PDW. PDW seems to be a very specific indicator of platelet activation, since it was not elevated during single platelet distention caused by platelet swelling. Thus, any factor declining PDW levels, may have beneficial effects on vaso-occlusive events. The aim of this basic study was to evaluate the effect of U-74389G in a rat model of HR using mean blood platelet distribution width (PDW) levels.

\section{Methods}

\section{Animal eligibility}

This animal research was licensed by the Veterinary Address of Agia Paraskevi based Prefecture. The number of licenses were those of 3693/12-11-2010 \& 14/10-1-2012. The required consumables, reagents and equipment needed were a grant of ELPEN Pharmaceuticals Co Inc S.A. at Pikermi, Attiki. The humane animal care adopted for Albino female Wistar rats included the normal housing of rats in laboratory 7 days before the experiment; probable required postexperimental euthanasia of the rats and the non stop oxygen supply, electrocardiogram and acidometry administration. Rats were randomly assigned to four experimental groups, each consisted by 10 animals. The following protocols of HR were applied in all of groups:

\section{1. $45 \mathrm{~min}$ hypoxia followed by $60 \mathrm{~min}$ reoxygenation for group $\mathrm{A}$}

2. 45 min hypoxia followed by 120 min reoxygenation for group $B$ 
3. 45 min hypoxia followed by immediate U-74389G intravenous (IV) administration and 60 min reoxygenation for group C

4. 45 min hypoxia followed by immediate U-74389G IV administration and 120 min reoxygenation for group D.

The U-74389G dose was $10 \mathrm{mg} / \mathrm{Kg}$ body mass of animals. The detailed anesthesiologic technique following prenarcosis, is described in related references. ${ }^{3-5}$ Then the protocol of HR was executed. Hypoxia was caused by 45 min clamping inferior aorta over renal arteries with forceps. Reoxygenation was restored by removing the clamp. The removal then restored the inferior aorta patency. U-74389G was administered exactly at the beginning of reoxygenation through the catheterized inferior vena cava. The PDW levels were determined at 60th min of reoxygenation (for $\mathrm{A}$ and $\mathrm{C}$ groups) and at 120th min of reoxygenation (for B and D groups). Fourty female rodents were used (mean weight 231. 875g (Std. Dev: 36.59703g), with minimum mass $165 \mathrm{~g}$ and maximum mass $320 \mathrm{~g}$. Rats' mass could be propably a confusing factor, e.g. more obese rats to have higher PDW levels. This assumption was also investigated.

\section{Control groups}

20 control rats (mean mass 252.5g (Std. Dev: 39.31988g)) eperienced 45 min hypoxia followed by reoxygenation.

Group A: Reoxygenation lasted for $60 \mathrm{~min}$ ( $\mathrm{n}=10$ controls rats) mean mass $243 \mathrm{~g}$ (Std. Dev: 45.77724g), mean PDW levels 16.06\% (Std. Dev: $0.2547331 \%$ ) (Table 2).

Group B: Reoxygenation lasted for $120 \mathrm{~min}$ ( $\mathrm{n}=10$ controls rats) mean mass 262g (Std. Dev: 31.10913g), mean PDW levels 15.83\% (Std. Dev: 0.2540778 \%) (Table 2).

Lazaroid (L) group. $20 \mathrm{~L}$ rats (mean mass 211.25g (Std. Dev: $17.53755 \mathrm{~g}$ ) experienced $45 \mathrm{~min}$ hypoxia followed by reoxygenation in the commencement of which $10 \mathrm{mg} \mathrm{U}-74389 \mathrm{G} / \mathrm{kg}$ body weight were IV administered.

Group C: Reoxygenation lasted for $60 \mathrm{~min}(\mathrm{n}=10 \mathrm{~L}$ rats) mean mass 212.5g (Std. Dev: 17.83411g), mean PDW levels 16.24\% (Std. Dev: $0.1807669 \%$ ) (Table 2).

Group D: Reoxygenation lasted for $120 \mathrm{~min}$ ( $\mathrm{n}=10 \mathrm{~L}$ rats) mean mass 210g (Std. Dev: 18.10463g), mean PDW levels 16.23\% (Std. Dev: $0.5774464 \%$ ) (Table 2).

\section{Statistical analysis}

Rats of each group were compared by weight and PDW level with each other by statistical paired t-tests (Table 3). Any probable significant difference among PDW levels, was searched whether it owed in potent significant mass correlations. The application of generalized linear models (glm) with dependant variable the PDW levels and independent variables the administration of U-74389G or no drug; the reoxygenation time and their combination was followed. Inserting the rats' mass also as an independent variable at glm analysis, a non significant relation resulted in $(\mathrm{p}=0.2303)$, so as to further investigation was not needed.

\section{Results}

The glm application resulted in: U-74389G administration kept significantly increased the PDW levels by $0.29 \%(0.0474504 \%$ $0.5325497 \%)(\mathrm{p}=0.0204)$. This finding was accordant with the results of paired t-test $(\mathrm{p}=0.0425)$. Reoxygenation time then, non-significantly decreased the PDW levels by $0.12 \%(-0.3775798 \%-0.1375794 \%)$ $(\mathrm{p}=0.3516)$, also accordant with paired t-test $(\mathrm{p}=0.3044)$. However, the combination of $\mathrm{U}-74389 \mathrm{G}$ administration and reoxygenation time together significantly increased the PDW levels by $0.1563636 \%$ $(0.007857 \%-0.3048701 \%)(\mathrm{p}=0.0396)$. Summing up the above and Table 3 , the Tables $4 \&$ Table 5 appear, concerning the restoring influence of U-74389G in connection with reoxygenation time.

Table I The U-74389G influence $( \pm S D)$ on the levels of some seric variables ${ }^{3}$ concerning reperfusion (rep) time

\begin{tabular}{|c|c|c|c|c|c|c|c|c|}
\hline Variable & Ih rep & p-value & I.5h rep & p-value & $2 \mathrm{~h}$ rep & p-value & $\begin{array}{l}\text { Interaction of } \\
\mathrm{U}-74389 \mathrm{G} \text { and rep }\end{array}$ & p-value \\
\hline$\overline{R B C}$ & $+1.39 \% \pm 0.71 \%$ & $0.716 I$ & 0.0096 & 0.8106 & $-.10 \% \pm 0.05 \%$ & 0.9762 & $+1.05 \% \pm 0.53 \%$ & 0.4911 \\
\hline hemoglobin & 0.08 & 0.0925 & 0.06 & 0.0604 & 0.059 & 0.3544 & 0.038 & 0.0423 \\
\hline $\begin{array}{l}\text { mean corpuscular } \\
\text { hemoglobin }\end{array}$ & $r_{0.0273}$ & 0.0663 & 0.0297 & 0.0001 & 0.0374 & 0.0003 & $1.33 \%+0.36 \%$ & 0.0005 \\
\hline platelet-crit & 0.1367 & 0.6373 & 0.1552 & 0.1064 & 0.2369 & 0.0833 & 0.1045 & 0.0712 \\
\hline Glucose & -0.0291 & 0.0663 & -0.0651 & 0.0001 & -0.0822 & 0.0003 & -0.0348 & 0.0005 \\
\hline total protein & -0.0249 & 0.0663 & -0.0558 & 0 & -0.0704 & 0 & -0.0298 & 0 \\
\hline $\begin{array}{l}\text { alkaline } \\
\text { phosphatase }\end{array}$ & $+22.66 \% \pm 12.37 \%$ & 0.0663 & 0.396 & 0.0001 & $0.508 I$ & 0.0003 & 0.2254 & 0.0005 \\
\hline $\begin{array}{l}\text { Creatine }^{5} \\
\text { phosphokinas }\end{array}$ & 0.6807 & 0.0012 & 0.5254 & 0.026 & $0.466 I$ & 0.4951 & 0.2796 & 0.077 \\
\hline Sodium & $+1.22 \% \pm 0.66 \%$ & 0.0707 & 0.0078 & $0.77 \mid 4$ & 0.0016 & 0.3995 & 0.0004 & 0.3693 \\
\hline Chloride & $-0.58 \% \pm 0.77 \%$ & 0.4533 & -0.0044 & 0.0879 & -0.006 & 0.1113 & -0.0037 & 0.0159 \\
\hline Calcium & $0 \% \pm 1.75 \%$ & I & 0.0096 & 0.8782 & 0.0126 & 0.8492 & $+0.14 \% \pm 0.64 \%$ & 0.8245 \\
\hline phosphorus & 0.0328 & 0.7966 & 0.0171 & 0.5789 & 0.0348 & 0.8129 & 0.0091 & 0.5771 \\
\hline magnesium & 0.0492 & 0.7033 & 0.0247 & 0.9171 & 0.0338 & 0.7161 & 0.0494 & 0.8228 \\
\hline Mean & $+5.92 \% \pm 16.16 \%$ & 0.3643 & $+4.97 \% \pm 13.48 \%$ & 0.3259 & $+4.03 \% \pm 13.50 \%$ & 0.3691 & 0.102 & 0.2532 \\
\hline
\end{tabular}

Table 2 Weight and mean platelet distribution width levels and Std. Dev. of groups

\begin{tabular}{llll}
\hline Groups & Variable & Mean & Std. Dev \\
\hline A & Weight & $243 \mathrm{~g}$ & $45.77724 \mathrm{~g}$ \\
& PDW & $16.06 \%$ & $0.2547331 \%$ \\
B & Weight & $262 \mathrm{~g}$ & $31.10913 \mathrm{~g}$ \\
& PDW & $15.83 \%$ & $0.2540778 \%$ \\
C & Weight & $212.5 \mathrm{~g}$ & $17.8341 \mathrm{~g}$ \\
& PDW & $16.24 \%$ & $0.1807669 \%$ \\
D & Weight & $210 \mathrm{~g}$ & $18.10463 \mathrm{~g}$ \\
& PDW & $16.23 \%$ & $0.5774464 \%$ \\
\hline
\end{tabular}


Table 3 Statistical significance of mean values difference for groups (DG) after statistical paired test application

\begin{tabular}{llll}
\hline DG & Variable & Difference & p-value \\
\hline A-B & Weight & $-19 \mathrm{~g}$ & 0.2423 \\
& PDW & $0.23 \%$ & 0.0394 \\
A-C & Weight & $30.5 \mathrm{~g}$ & 0.0674 \\
& PDW & $-0.18 \%$ & 0.2789 \\
A-D & Weight & $33 \mathrm{~g}$ & 0.0574 \\
& PDW & $-0.17 \%$ & 0.4307 \\
B-C & Weight & $49.5 \mathrm{~g}$ & 0.0019 \\
& PDW & $-0.41 \%$ & 0.0076 \\
B-D & Weight & $52 \mathrm{~g}$ & 0.0004 \\
& PDW & $-0.40 \%$ & 0.1012 \\
C-D & Weight & $2.5 \mathrm{~g}$ & 0.7043 \\
& PDW & $0.01 \%$ & 0.9625 \\
\hline
\end{tabular}

Table 4 Statistical significance of mean values difference for groups (DG) after statistical paired t test application

\begin{tabular}{lllll}
\hline & & Reperfusion time & \multicolumn{2}{c}{ p-values } \\
Increase & $\mathbf{9 5 \%}$ c. in. & t-test & 0.2789 & 0.1948 \\
\hline$+0.18 \%$ & $-0.1008224 \%-0.4608226 \%$ & $\mathrm{Ih}$ & 0.0425 & 0.0204 \\
$+0.29 \%$ & $0.0474504 \%-0.5325497 \%$ & $\mathrm{I} .5 \mathrm{~h}$ & 0.1012 & 0.0602 \\
$+0.4 \%$ & $-0.0191327 \%-0.8191325 \%$ & $2 \mathrm{~h}$ & 0.3044 \\
$-0.12 \%$ & $-0.3775798 \%-0.1375794 \%$ & reoxygenation time & 0.3516 \\
$+0.1563636 \%$ & $0.007857 \%-0.3048701 \%$ & interaction & 0.0396 \\
\hline
\end{tabular}

Table 5 The (\%) increasing influence of U-74389G in connection with reperfusion time

\begin{tabular}{llll}
\hline Increase & +SD & Reperfusion time & p-values \\
\hline$+1.10 \%$ & $+0.88 \%$ & $\mathrm{Ih}$ & 0.2368 \\
$+1.79 \%$ & $+0.76 \%$ & $\mathrm{I} .5 \mathrm{~h}$ & $0.03 \mathrm{I}$ \\
$+2.49 \%$ & $+1.33 \%$ & $2 \mathrm{~h}$ & 0.0807 \\
$-0.745 \%$ & $+0.8 \mathrm{I} \%$ & reoxygenation time & 0.3280 \\
$+0.96 \%$ & $+0.46 \%$ & Interaction & 0.0396 \\
\hline
\end{tabular}

\section{Discussion}

The following cases show how hypoxia can influence PDW levels. Muscari et al. ${ }^{7}$ determined PDW values in elderly patients with coronary heart disease or at risk for stroke and associated it with subcutaneous abdominal fat $(p=0.02)$, fasting blood glucose $(p=0.002)$, prevalence of ischemic ECG changes $(p=0.004)$, prevalence of hepatic steatosis $(\mathrm{p}=0.07)$ and higher homeostasis Model Assessment (HOMA) index $(\mathrm{p}=0.09)$. Ihara et al. ${ }^{8}$ correlated TAT and VWF: Ag significantly associated positively with PDW levels between ischemic heart patients (IHD) and aged controls. Ihara et al. ${ }^{9}$ found PDW levels significantly higher in negatively imaged group than in positive one in match case- control ones $(\mathrm{p}=0.00461 \& 0.0146$, respectively) depicting the underlying pathophysiologic mechanism. Dudley et al. ${ }^{10}$ assessed scored elevated PDW levels (greater than 2 SD from mean), or presence of clinical ischemia at the outset for distinguishing correct diagnosis of primary thrombocythemia from reactive thrombocytosis (predictive value 89\%).

Literature showing how U-74389G can influence platelet count and their function do not exist. However, Pratt ${ }^{11}$ studied the effects of U-74389G on the pathophysiology of random skin flap necrosis or survival in pig model. Mechanisms of skin flap failure included among other, the alteration of platelet function with resultant accumulation of dangerous oxygen-free radicals. Random skin flap survival time was significantly improved after U-74389G administration. Platelet indices are probably the most ignored among all parameters by clinical laboratories. It is due to the difficulty of standardization, as indices are affected by a range of methodological problems, like that each laboratory determines its own reference intervals. Among all platelet indices, PDW is the most useful for distinguishing between reactive thrombocytosis and thrombocytosis associated with myeloproliferative disorder. Farias et al. ${ }^{12}$ recognized that determination of the PDW reference range is fundamental for the diagnosis and differentiation of several pathologies. Akpinar et al. ${ }^{13}$ found that furthermore increased PDW may be a useful predictor of slow coronary flow (SCF) patients due to impaired cell deformability. Ceylan et al. ${ }^{14}$ determined the severity of liver fibrosis in patients with chronic HBV infection using PDW as an independent variable among other markers. Alyamac Dizdar et al. ${ }^{15}$ associated patent ductus arteriosus with high PDW in preterm infants. Santimone et al. ${ }^{16}$ related PDW levels with inflammation. Zhang et al. ${ }^{17}$ applied the PDW level in basic differential diagnosis of thrombocytosis-related diseases: reactive thrombocytosis, chronic myeloproliferative disease including chronic myeloid leukemia, essential thrombocythemia and polycythemia vera. Boos et al. ${ }^{18}$ calculated a stepwise increase in PDW level as marker of platelet shape change, with increasing severity of complicating hypertensive disease. Jurcuţ et al. ${ }^{19}$ associated high PDW values with a higher number of affected coronary arteries: high vWF and low brachial flow-mediated dilation worse short-term prognosis in unstable angina patients. Wasiluk et al. ${ }^{20}$ considered an increased PDW vital in diagnosis of hemostatic disorders dysfunction in preterm newborns. Indraccolo et al. ${ }^{21}$ conservatively managed HELLP syndrome by PDW tests. Ravindran et al. ${ }^{22}$ predicted prothrombotic tendency in coronary artery disease patients by high PDW levels. Hyperlipidemia increases the reactivity of platelets in lipid content. This may accelerate atherogenesis and organized thrombogenesis on vascular surfaces. Chepurnov et al. ${ }^{23}$ supposed that modification of the virus in the course of adaptation to the host results in the appearance 
of properties boosting the enzymatic processes and hence, in depletion and failure of antioxidant and hemostatic defence, which aggravates the pathological process after inoculation of Ebola virus in guinea pigs. Nearly all authors associate elevating PDW levels induced or not with ominous diseases evolutions.

Early diagnosis of progressive activation of coagulation can help manage these diseases successfully. ${ }^{24}$ Reliable markers have been investigated recently, concerning activation of coagulation, such as prothrombin fragment $1+2$, thrombin-antithrombin complex (TAT), and platelet activation. Markers such as $\beta$-thromboglobulin ( $\beta$-TG) or soluble platelet $\mathrm{P}$-selectin was based on the fact that platelet activation causes morphologic changes of platelets, including both the spherical shape and pseudopodia formation. Platelets with increased number and size of pseudopodia differ in size, possibly affecting PDW. Mean values of PDW were significantly higher in patients with asserted platelet activation compared with healthy persons.

PDW is easily measured platelet index, which increases during platelet activation. Platelets change in shape during activation in order to obtain a larger surface. Their shape changes from discoid to spherical. Pseudopodia are formed as well. Therefore activated platelets seem larger. PDW was higher in subgroups of certain platelet activation. The increase of PDW was ascribed to platelet anisocytosis, which results from pseudopodia formation. The same observation was made in patients with ischemic heart disease and in vaso-occlusive crisis in sickle cell disease. Megakaryote hyperplasia was concluded that was responsible for PDW increase. However, coagulation is activated in vaso-occlusive crisis, possibly causing this increase of PDW.

On the contrary, large platelets did not show more intense activation in aggregometer. Indeed, large and variable in size platelets can be observed in several clinical conditions, such as hemorrhage and myeloproliferative disorders, without simultaneous activation of coagulation. Moreover, platelets shape and volume are variable, even in healthy persons. Thus, simultaneous increase of mean platelet volume (MPV) and PDW could reveal platelet activation than their serial measurements. PDW seems to be a very specific indicator of platelet activation, since it was not elevated during single platelet distention caused by platelet swelling. The combined use of MPV and PDW could predict activation of coagulation more efficiently.

Along, decrease in PDW over storage time was found. There was no pseudopodia formation during storage of blood samples could be concluded. Consequently, there was no platelet activation, except from single platelet distention and swelling. A decreased effect of storage time on PDW was also observed. Regarding the influence of time, it was shown that during sequential hourly measurements, there is an $88.34 \%$ possibility to detect a difference among the measurements.

\section{Conclusion}

U-74389G administration whether it interacted or not with reoxygenation time, borderline declined from significant to non significant the PDW levels, within short-time study period of 2 hours. This finding has huge clinical interest in clinical coagulopathy. A longer study time or a higher U-74389 dose may reveal more effective results in thromboembolic diseases.

\section{Acknowledgments}

This study was funded by Scholarship by the Experimental Research Center ELPEN Pharmaceuticals (E.R.C.E), Athens, Greece. The research facilities for this project were provided by the aforementioned institution.

\section{Conflicts of interst}

None.

\section{References}

1. https://www.caymanchem.com/app/template/Product.vm/catalog/75860

2. Shi F, Cavitt J, Audus KL 21-aminosteroid and 2-(aminomethyl) chromans inhibition of arachidonic acid-induced lipid peroxidation and permeability enhancement in bovine brain microvessel endothelial cell monolayers. Free Radic Biol Med. 1995;19(3):349-357.

3. Tsompos C, Panoulis C, Toutouzas K, et al. The effect of the antioxidant drug U-74389G on Magnesium levels during hypoxia-reoxygenation injury in rats. Annals of the Russian Academy of Medical Sciences. 2015;70(4):408-412. DOI:10.15690/vramn.v70.i4.1405.

4. Tsompos C, Panoulis C, Toutouzas K, et al.The acute effect of the antioxidant drug "U-74389G" on platelet-crit levels during hypoxia reoxygenation injury in rats. Acta Phlebologica. 2015;16(2):77-81.

5. Tsompos C, Panoulis C, Toutouzas K, et al. The Effect of the Antioxidant Drug "U-74389G" on Creatine Phosphokinase Levels during Ischemia Reperfusion Injury in Rats. Erciyes Med J. 2015;37(3):91-97.

6. Reis F, Parada B, Teixeira de Lemos E, et al. Hypertension induced by immunosuppressive drugs: a comparative analysis between sirolimus and cyclosporine. Transplant Proc. 2009;41(3):868-873.

7. Muscari A, De Pascalis S, Cenni A, et al. Determinants of mean platelet volume (MPV) in an elderly population: relevance of body fat, blood glucose and ischaemic electrocardiographic changes. Thromb Haemost. 2008;99(6):1079-1084.

8. Ihara A, Kawamoto T, Matsumoto K, et al. Relationship between hemostatic factors and the platelet index in patients with ischemic heart disease. Pathophysiol Haemost Thromb. 2006;35(5):388-391.

9. Ihara A, Kawamoto T, Matsumoto K, et al. Relationship between platelet indexes and coronary angiographic findings in patients with ischemic heart disease. Pathophysiol Haemost Thromb. 2006;35(5):376-379.

10. Dudley JM, Messinezy M, Eridani S, et al. Primary thrombocythaemia: diagnostic criteria and a simple scoring system for positive diagnosis. $\mathrm{Br}$ J Haematol. 1989;71(3):331-335.

11. Pratt MF Edmund Prince Fowler Award Thesis. Evaluation of random skin flap survival in a porcine model. Laryngoscope. 1996;106(6):700 712 .

12. Farias MG, Schunck EG, Dal Bó S, Definition of reference ranges for the platelet distribution width (PDW): a local need. Clin Chem Lab Med. 2010;48(2):255-257.

13. Akpinar I, Sayin MR, Gursoy YC, et al. Plateletcrit and red cell distribution width are independent predictors of the slow coronary flow phenomenon. J Cardiol. 2013;63(2):112-118.

14. Ceylan B, Mete B, Fincanci M, et al. A new model using platelet indices to predict liver fibrosis in patients with chronic hepatitis B infection. Wien Klin Wochenschr. 2013;125(15-16):453-460.

15. Alyamac Dizdar E, Ozdemir R, Nur Sari F, et al. Low platelet count is associated with ductus arteriosus patency in preterm newborns. Early Hum Dev. 2012;88(10):813-816.

16. Santimone I, Di Castelnuovo A, De Curtis A, et al. White blood cell count, sex and age are major determinants of heterogeneity of platelet indices in an adult general population: results from the MOLI-SANI project. Haematologica. 2011;96(8):1180-1188.

17. Zhang KJ, Lu QY, Li P, et al. Significance of platelet parameters and lactate dehydrogenase level in differential diagnosis for thrombocytosis. Zhongguo Shi Yan Xue Ye Xue Za Zhi. 2010;18(4):972-975. [Article in Chinese]. 
18. Boos CJ, Beevers GD, Lip GY Assessment of platelet activation indices using the ADVIATM 120 amongst 'high-risk' patients with hypertension. Ann Med. 2007;39(1):72-78.

19. Jurcut R, Arsenescu I, Puşcariu T, et al. Is interleukin-18 correlated with endothelial dysfunction and platelet activation in patients with unstable angina? Rom J Intern Med. 2005;43(3-4):199-209.

20. Wasiluk A, Osada J, Dabrowska M, et al. Does prematurity affect platelet indices? Adv Med Sci. 2009;54(2):253-255.

21. Indraccolo U, Gentile G, Manfreda VM, et al. The development of disseminated intravascular coagulation in hemolysis, elevated liver enzymes, and low platelet count syndrome (HELLP) at very early gestational age. Minerva Ginecol. 2008;60(5):445-450.
22. Ravindran R, Krishnan LK. Increased platelet cholesterol and decreased percentage volume of platelets as a secondary risk factor for coronary artery disease. Pathophysiol Haemost Thromb. 2007;36(1):45-51.

23. Chepurnov AA, Dadaeva AA, Zhukov VA, et al. Change in biochemical and hemostatic indicators in guinea pigs upon administering Ebola virus preparations. Vopr Virusol. 1997;42(4):171-175.[Article in Russian].

24. Vagdatli E, Gounari E, Lazaridou E, et al. Platelet distribution width: a simple, practical and specific marker of activation of coagulation. Hippokratia. 2010;14(1):28-32. 